\title{
Method for conducting in situ high- temperature digital image correlation with simultaneous synchrotron measurements under thermomechanical conditions
}

Cite as: Rev. Sci. Instrum. 91, 033705 (2020); https://doi.org/10.1063/1.5124496

Submitted: 14 August 2019. Accepted: 15 February 2020 . Published Online: 05 March 2020

Lin Rossmann (D), Brooke Sarley, Johnathan Hernandez, Peter Kenesei, Alain Köster, Janine Wischek, Jonathan Almer, Vincent Maurel, Marion Bartsch, and Seetha Raghavan (D)

Eriew Online




\title{
Method for conducting in situ high-temperature digital image correlation with simultaneous synchrotron measurements under thermomechanical conditions
}

\author{
Cite as: Rev. Sci. Instrum. 91, 033705 (2020); doi: 10.1063/1.5124496 \\ Submitted: 14 August 2019 - Accepted: 15 February 2020 • \\ Published Online: 5 March 2020
}

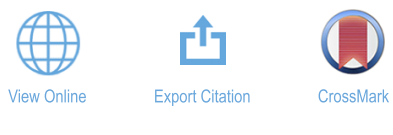

Lin Rossmann, ${ }^{1}$ (D) Brooke Sarley, ${ }^{2}$ Johnathan Hernandez, ${ }^{2}$ Peter Kenesei, ${ }^{3}$ Alain Köster, ${ }^{4}$ Janine Wischek, Jonathan Almer, ${ }^{3}$ Vincent Maurel, ${ }^{4}$ Marion Bartsch, ${ }^{5}$ and Seetha Raghavan ${ }^{2, a)}$ (iD

\begin{abstract}
AFFILIATIONS
${ }^{1}$ Department of Materials Science and Engineering, University of Central Florida, Orlando, Florida 32816, USA

${ }^{2}$ Department of Mechanical and Aerospace Engineering, University of Central Florida, Orlando, Florida 32816, USA

${ }^{3}$ X-Ray Science Division, Advanced Photon Source, Argonne National Laboratory, Argonne, Illinois 60439, USA

“MINES ParisTech, PSL Research University, MAT - Centre des Matériaux, 91000 Évry, France

${ }^{5}$ German Aerospace Center, Institute of Materials Research, Linder Höhe, Köln 51147, Germany
\end{abstract}

a) Author to whom correspondence should be addressed: seetha.raghavan@ucf.edu

\begin{abstract}
This work presents a novel method of obtaining in situ strain measurements at high temperature by simultaneous digital image correlation (DIC), which provides the total strain on the specimen surface, and synchrotron x-ray diffraction (XRD), which provides lattice strains of crystalline materials. DIC at high temperature requires specialized techniques to overcome the effects of increased blackbody radiation that would otherwise overexpose the images. The technique presented herein is unique in that it can be used with a sample enclosed in an infrared heater, remotely and simultaneously with synchrotron XRD measurements. The heater included a window for camera access, and the light of the heater lamps is used as illumination. High-temperature paint is used to apply a random speckle pattern to the sample to allow the tracking of displacements and the calculation of the DIC strains. An inexpensive blue theatrical gel filter is used to block interfering visible and infrared light at high temperatures. This technique successfully produces properly exposed images at $870{ }^{\circ} \mathrm{C}$ and is expected to perform similarly at higher temperatures. The average strains measured by DIC were validated by an analytical calculation of the theoretical strain. Simultaneous DIC and XRD strain measurements of Inconel 718 (IN718) tensile test specimens were performed under thermal and mechanical loads and evaluated. This approach uses the fact that with DIC, the total strain is measured, including plastic strain, while with XRD, only elastic strain is captured. The observed differences were discussed with respect to the effective deformation mechanisms.
\end{abstract}

Published under license by AIP Publishing. https://doi.org/10.1063/1.5124496

\section{INTRODUCTION}

In situ experimentation is a highly effective approach to investigate material behavior under extreme conditions. However, by nature of the method, the opportunity to make meaningful observations is fleeting and should be maximized. The instrumentation presented in this work combines two in situ observation methods, applied simultaneously, to observe the deformation of the material under thermomechanical load on the micro- to nano-scales. The applied techniques were synchrotron $\mathrm{x}$-ray diffraction (XRD), which captures the changes in lattice parameters of crystalline materials due to thermal expansion and elastic deformation, and digital image correlation (DIC), which provides the total strain on a specimen surface. Comparing strain results from both methods is a unique approach to investigate acting deformation mechanisms and anisotropic behavior. 


\section{A. Observing strains with both DIC and $x$ rays}

This work presents a method for simultaneously measuring a material's lattice strain and total strain under thermomechanical conditions. Observing dual strains has a wide range of applications, including ceramics and composites; in this work, the instrumentation is demonstrated with a study of an additively manufactured superalloy. Inconel 718 is a nickel-based superalloy used in high-temperature aerospace applications, and additive manufacturing (AM) provides new opportunities such as more complex geometries and fewer welds and fasteners. However, the effect of the AM process on the material's microstructure and resultant mechanical properties must be understood before widespread adoption. The thermal gradient during the process creates preferred orientation in the material's crystal structure, resulting in anisotropic properties and behavior, in some cases metastable phases being formed. Simultaneously observing total strain and strains in the different planes of the crystal lattice offers new insight into these effects.

Total strain is captured by digital image correlation (DIC), in which a speckle pattern applied to the sample is photographed during load, and software tracks the movements of the speckles with respect to a reference image to compute the strain field. This strain, therefore, includes any plastic strain as well as elastic strain. Lattice strains are captured using high-energy $x$-ray diffraction (XRD) at the Advanced Photon Source (APS) 1-ID beamline at Argonne National Laboratory. Synchrotron XRD is a powerful method that enables fast measurements of a transmission diffraction pattern on a detector. With a $2 \mathrm{D}$ image of the transmitted scattered $\mathrm{x}$ rays, the atomic structure including lattice strains can be observed in more than one direction, helpful for studying anisotropy. The high-speed capability of the APS enables in situ observation of microstructural behavior under any condition that can be applied at the beamline. In this work, XRD and DIC were conducted, while the sample was mechanically and thermally loaded by mounting the sample in a load frame coupled with an infrared heater. A schematic of the simultaneous acquisition of DIC and XRD strain is shown in Fig. 1.

\section{B. Current methods of high-temperature DIC}

DIC at room temperature is well established ${ }^{1,2}$ and commonly performed, ${ }^{3}$ but at high temperatures, it presents additional challenges. At high temperatures, increased black body radiation causes the image to become overexposed, despite fast shutter speed, small aperture, and low brightness level setting. This overexposure obscures the applied speckle pattern, making displacement and strain calculations much less accurate and sometimes impossible.

Other researchers have used various methods to obtain DIC images at high temperatures. ${ }^{4-14}$ One method is to illuminate the sample with laser light, which has a sufficient power density to outweigh the thermal radiation; DIC images at $1200^{\circ} \mathrm{C}$ have been obtained this way. ${ }^{5}$ A commonly used technique is to illuminate the

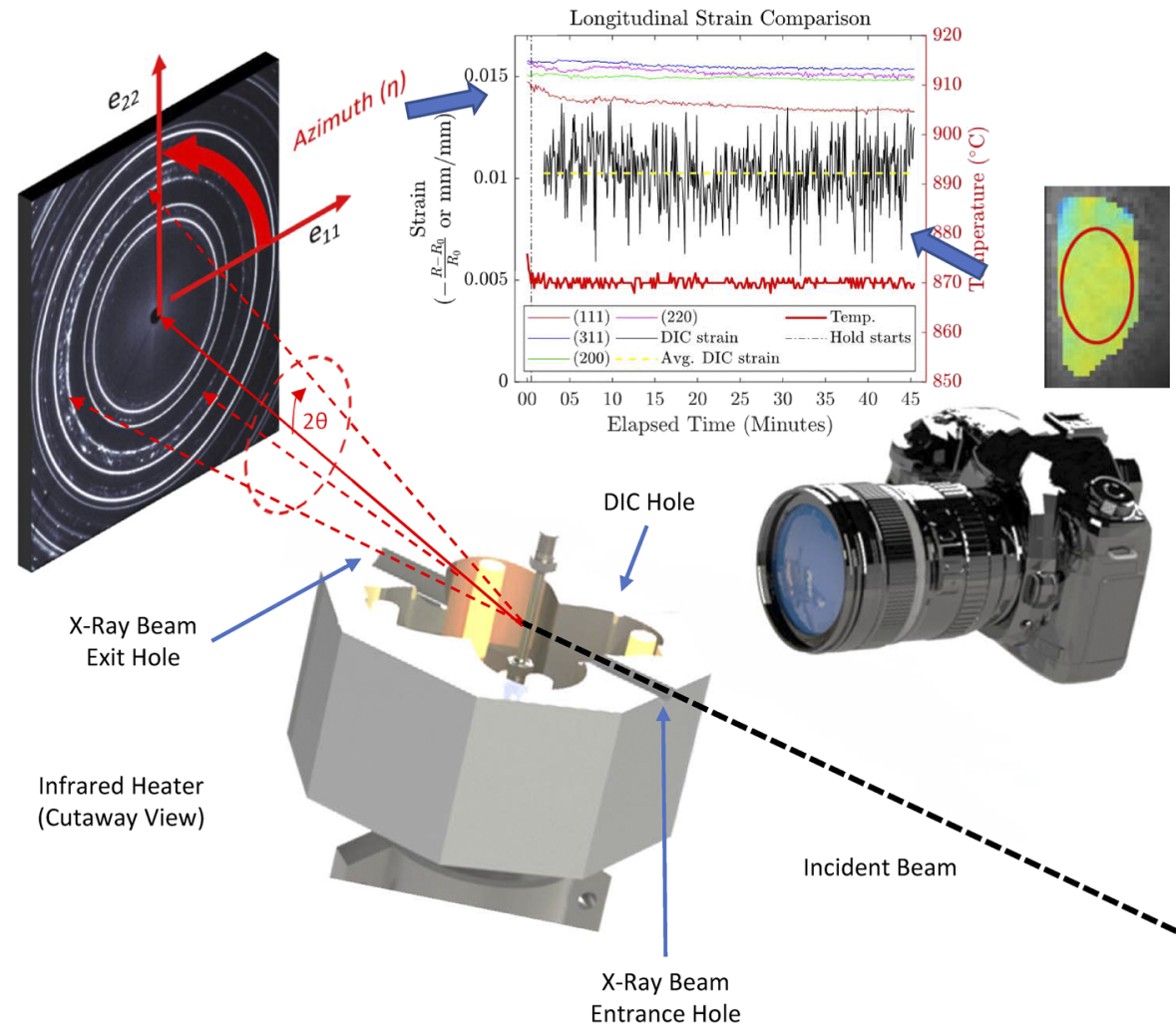

FIG. 1. Simultaneous acquisition of lattice strains via synchrotron XRD and total strain via DIC enables them to be directly compared, providing insight into the lattice orientation dependency of mechanical properties. These measurements have been done in situ with an infrared heater and a load frame. The figure displays a cutaway view of the heater with the specimen in the focus line of four concentrating elliptical mirrors. 

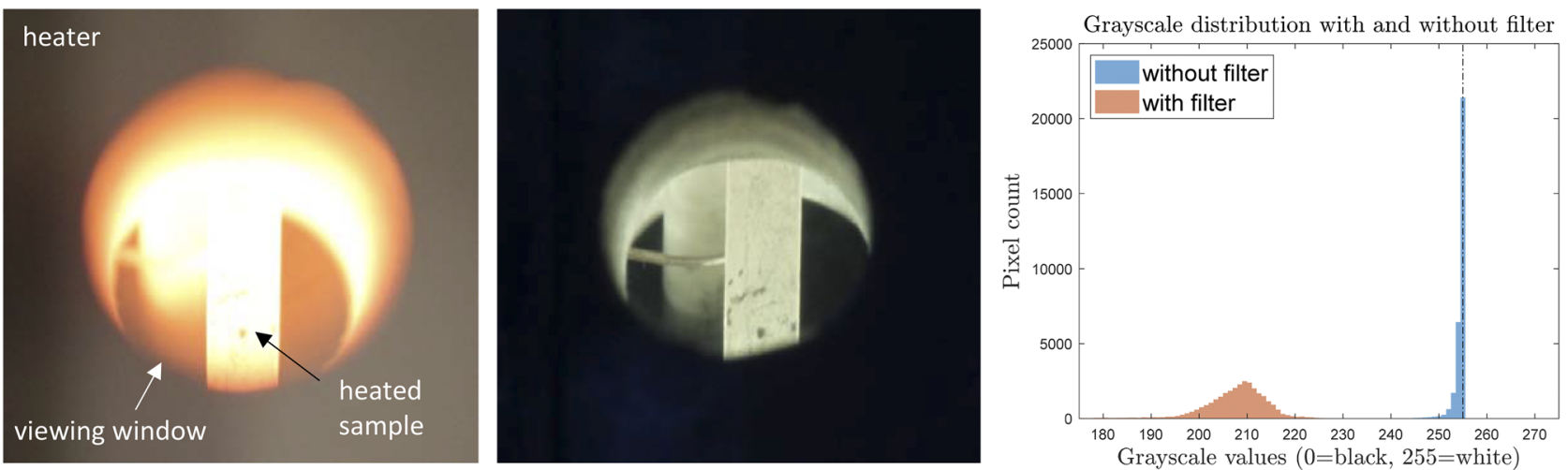

FIG. 2. Steel rod heated to $870^{\circ} \mathrm{C}$, photographed without a filter (left) and with a filter (center), with grayscale histograms of the sample (right). The histograms were made from the photos cropped to only the area of the sample. The $x$-axis values are grayscale values from 0 (black) to 255 (white), but the $x$-axis is shown from 175 to 275 for greater visibility and legibility, especially regarding the saturation spike at 255 .

sample with monochromatic light in the blue or UV wavelength range and use a corresponding bandpass filter on the lens. ${ }^{9,15-22}$ Blue lighting with a matching bandpass filter was used to photograph woven $\mathrm{Si} / \mathrm{SiC}$ composites at $1550{ }^{\circ} \mathrm{C}$ in a custom quartz lamp heating device, ${ }^{20}$ and ultraviolet lighting and filters were used to acquire images of thermal barrier coatings in an optical microscope heating stage at $1200^{\circ} \mathrm{C} .^{10}$ Carbon fibers in an induction heater were imaged at $2600^{\circ} \mathrm{C}$ using a bandpass filter, neutral density filters, and polarizing filters. ${ }^{18}$ These approaches, while effective, cannot be used when the sample is enclosed in an infrared heater.

\section{Use of heater lamps with blue filter}

The method presented in this work does not require additional lighting and can be used with samples enclosed in an infrared heater, overcoming a limitation of methods described previously. The sample is illuminated by the lamps of the infrared heater, rather than external lighting equipment. To eliminate excess thermal radiation, a blue theatrical gel filter is placed on the camera lens; this filter is inexpensive and can be easily cut to fit a lens filter adapter. The chosen filter is one of a large catalog of theatrical gels and was chosen to best suit the particular heater; transmission percentage per wavelength data are available to aid selection. No bandpass, neutral density, or polarizing filters are required, reducing the complexity of the experimental setup. The effect of the filter is shown in Fig. 2. The filter blocks orange, red, and infrared light, producing a properly exposed image. Without the filter and using a similar exposure time (1/80 s without filter and 1/50 s with filter), the image is overexposed (has white regions with no visual detail). The accompanying histograms show the grayscale distribution of the two images, cropped to the sample region used for DIC. The image without the filter is almost completely saturated, indicating pure white with no contrast or image detail. It is possible that a properly exposed image could have been achieved without the filter using a sufficiently fast shutter speed, but the filter makes proper exposure much easier to achieve.

\section{EXPERIMENTAL METHODS}

\section{A. Sample manufacturing and preparation}

Experiments were performed using cylindrical dogbone tensile specimens. Three rods of IN718 were manufactured via selective laser melting. They were then machined to a gage diameter of $5 \mathrm{~mm}$ and a measurement length of $10 \mathrm{~mm}$. Paint, applied using an air gun or a spray can, is one of the most common methods of creating unique local patterns for DIC software to track. ${ }^{23}$ Hightemperature paint was applied to a region of the surface of each sample as follows. Masking tape was applied to each sample to expose only a rectangular region measuring $3 \mathrm{~mm} \times 19 \mathrm{~mm}$. Because the high-temperature paint contains ceramic particles, the painted region must be kept out of the path of the synchrotron beam lest it creates additional diffraction patterns; this restriction prevents the DIC and XRD measurements from having the same view of the sample. Multiple light coats of flat white VHT Flameproof ${ }^{\mathrm{TM}}$ hightemperature spray paint were applied to the samples and allowed to fully dry, creating a uniform white region. The white background provides improved contrast and eliminates glare. One coat of black VHT Flameproof spray paint was applied to the samples with the spray can held farther away, resulting in a fine mist-like speckle pattern. After the paint was fully dry, the masking tape was removed. Figure 3 shows the resulting pattern applied to a sample.

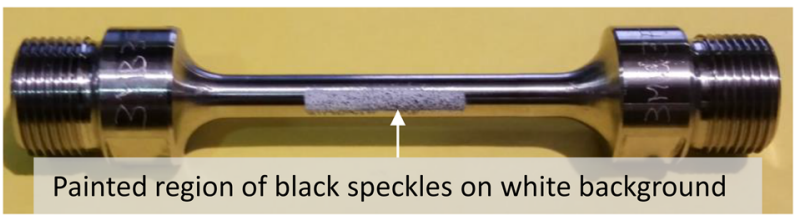

FIG. 3. Dogbone tensile specimen of SLM IN718 with speckle pattern for DIC. The white paint increases contrast and eliminates glare, and the black paint provides the speckles for tracking displacement. 


\section{B. Experimental setup}

A Precision Controls E4 High-temp Infrared Chamber Heater and Control System (Precision Control Systems, Inc., Eden Prairie, $\mathrm{MN}$ ) was modified to introduce entrance and exit holes for the synchrotron $\mathrm{x}$-ray beam in previous works; ${ }^{24,25}$ using high-energy $\mathrm{x}$ rays enables the use of a relatively small ( 1 in. diameter) exit hole, thanks to the small Bragg angle. A $4 \mathrm{~mm}$ hole was also introduced to serve as a window for the camera. The heater system includes a hinged clam-shell style heating chamber containing four polished aluminum water-cooled reflectors to focus the infrared (IR) energy of four $2 \mathrm{~kW}$ lamps on the sample located in the center. The viewing hole diameter was chosen to be the largest possible without interfering with the lamps and other components of the heater. The sample was positioned such that its painted region was parallel to the incident and diffracted $\mathrm{x}$ rays to prevent the beam from striking the paint.

The sample was mounted into the load frame while inside the heater such that the heater, load frame, and sample translated together with respect to the stationary $\mathrm{x}$-ray beam to move the measurement location on the sample. Due to the load frame and other equipment, a standard tripod could not be used to support the camera. A variety of adapters were used with an optical table with threaded holes to create an adjustable mounting system for the camera. During sample mounting, care was taken to align the painted region facing the viewing hole. The camera was mounted on a pole secured to the optical table on which the load frame was mounted, ensuring that when the sample is moved with respect to the beam, the sample-to-camera distance is not changed. A schematic of the setup is shown in Fig. 1.

Photographs of the setup are shown in Fig. 4, showing the position of the camera and heater. An S-type thermocouple was looped around the sample and threaded through the beam entrance hole.

Before data acquisition for each of the samples, the heater was held at $200{ }^{\circ} \mathrm{C}$ to provide illumination from the heater lamps for focusing the camera. After focusing, a tensile load of $0.98 \mathrm{kN}$ was applied and held constant. After the tensile load was applied, the temperature was increased to the target temperature and held constant during measurement acquisition.

The three samples were each held at the same mechanical load and different temperature holds. In this work, only the results from the sample held at $870{ }^{\circ} \mathrm{C}$ are presented for brevity.

\section{In situ synchrotron studies}

$\mathrm{X}$-ray diffraction patterns were obtained using the high-energy synchrotron X-ray diffraction instrument offered at the 1-ID Beamline of the APS at ANL. This high-energy XRD technique employs a monochromatic $\mathrm{X}$-ray beam in transmission geometry with a twodimensional flat-panel area detector that collects the shape of the Debye-Scherrer rings, capable of high-resolution measurements of lattice strain in the bulk polycrystalline material. ${ }^{26} \mathrm{~A}$ beam with an energy of $90.532 \mathrm{keV}$ and a size of $100 \mu \mathrm{m} \times 100 \mu \mathrm{m}$ was used to achieve sufficient transmission through the center of the test section. Biaxial strain measurements were resolved into longitudinal $\left(\varepsilon_{y y}\right)$ and transverse $\left(\varepsilon_{x x}\right)$ directions, ${ }^{25,27-29}$ as shown in Fig. 5.

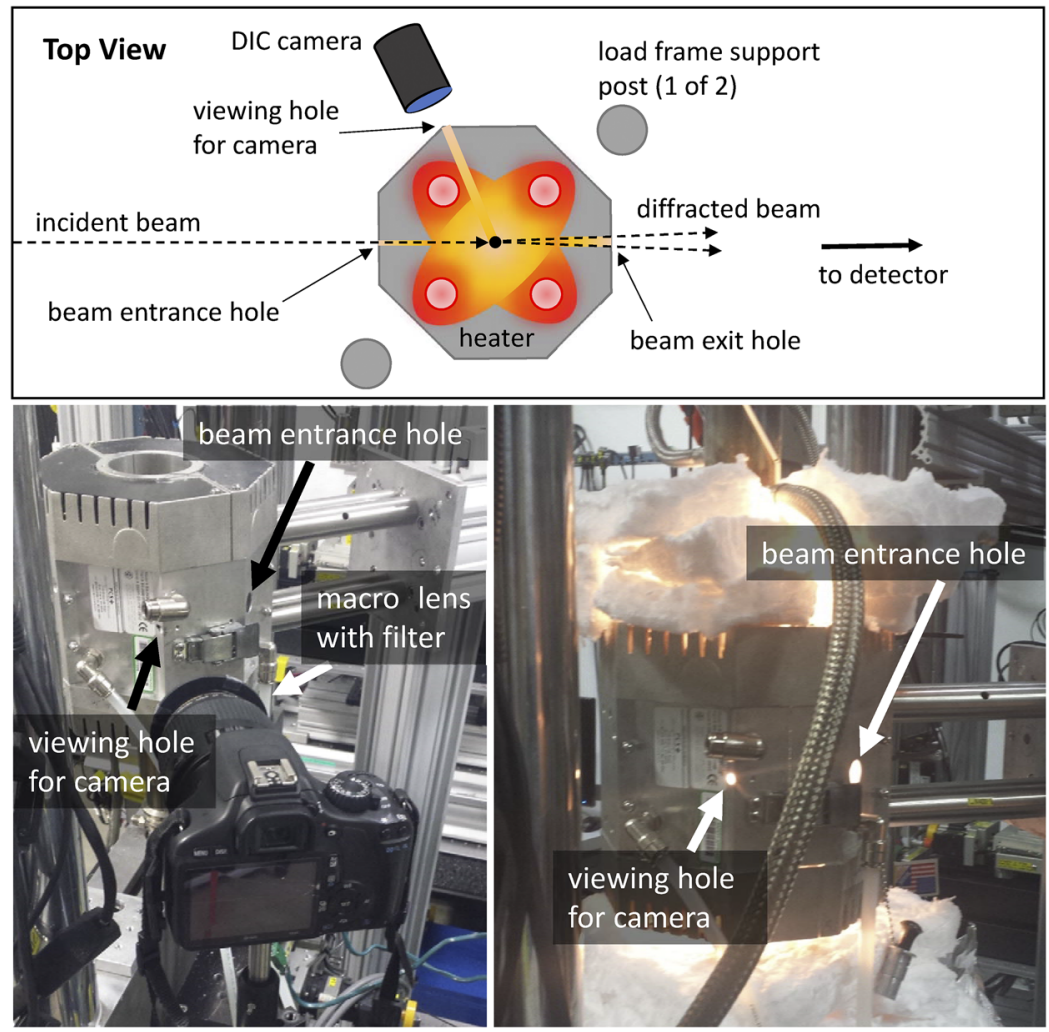

FIG. 4. A camera with a macro-lens and blue filter is mounted to an optical table facing a viewing hole in a clamshell-style infrared heater, which is mounted to the same optical table. The viewing hole and the entrance hole for the synchrotron beam are more easily seen when the heater lamps are on (right). The heater lamps provide the illumination for photographing the sample. 


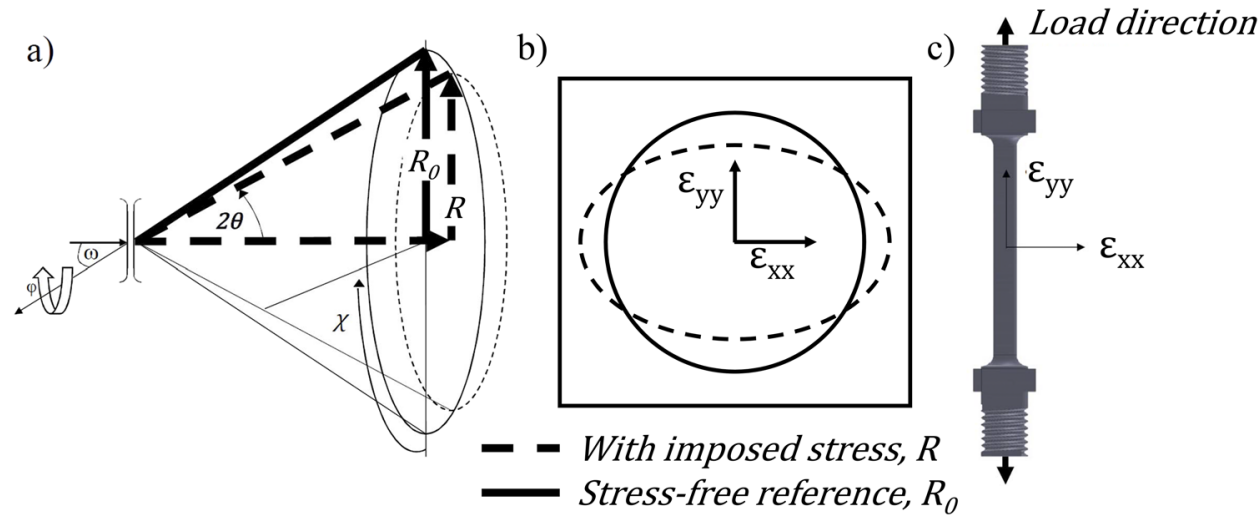

FIG. 5. Schematics of transmission $x-$ ray diffraction geometry (a), 2D diffraction image bi-axial strain analysis (b), and lattice strain direction relationship to orientation of the sample (c)
The average error for the strain of each lattice plane (the standard deviation of the measured strain around the azimuth of the diffraction ring from the least-squares curve fit around the azimuth) is listed in Table I. For precise and accurate measurements, calibration of the detector position and orientation with respect to the incident beam was first performed using ceria powder. Stress-free reference ring radii $\left(\mathrm{R}_{0}\right)$ were taken from a sample at room temperature with no applied load. XRD measurements were taken every $10 \mathrm{~s}$ during the high-temperature hold.

\section{DIC capture and analysis}

A Canon ${ }^{\circledR}$ T2i Rebel ${ }^{\circledR}$ 18-megapixel digital single-lens-reflex camera was used with a Canon Macro-EF $100 \mathrm{~mm} \mathrm{f/2.8} \mathrm{lens.} \mathrm{A}$ Roscolux $^{\mathrm{TM}}$ No. 72 blue theatrical gel was cut into a circle and fit into a lens adapter, which was mounted to the lens. The camera was operated remotely using the Canon EOS Utility ${ }^{\circledR}$ software, with a mini-universal serial bus (USB) to USB cable and a USB extension cable connecting the camera and a laptop computer. The USB extension cable was threaded through a cable slot in the wall of the experimental hutch, allowing the camera to be operated from the laptop computer outside the hutch. When operated through the EOS Utility software, the images are stored on the connected computer, so filling the camera's storage card during the experiment was not a concern. This capability is essential when conducting synchrotron measurements as there can be no access to the experimental hutch while measurements are in progress. The camera's clock was also synchronized to that of the XRD area detector.

Focusing was done for each sample tested. To have enough light to focus, the heater was programmed to hold at $200^{\circ} \mathrm{C}$. The lens

TABLE I. Average error in calculated strain per lattice plane.

\begin{tabular}{lcc}
\hline \hline Lattice plane & Transverse & Longitudinal \\
\hline$(111)$ & $9.45 \times 10^{-5}$ & $9.64 \times 10^{-5}$ \\
$(200)$ & $1.04 \times 10^{-4}$ & $2.11 \times 10^{-4}$ \\
$(220)$ & $7.71 \times 10^{-5}$ & $9.80 \times 10^{-5}$ \\
$(311)$ & $6.86 \times 10^{-5}$ & $6.65 \times 10^{-5}$ \\
Avg. & $8.61 \times 10^{-5}$ & $1.18 \times 10^{-4}$ \\
\hline
\end{tabular}

was then manually focused on the speckle pattern visible through the viewing hole. The shooting mode was set to aperture preferred, the mode in which the aperture is selected by the user, and the shutter speed is automatically adjusted by the camera to produce a properly exposed image. During the ramp up to the target hold temperature, test images were taken to determine whether exposure compensation was required; between $-12 / 3$ and -2 , stops of compensation were used for the samples. Adjustment of capture settings continued for a few minutes after the hold temperature was reached, which is why the DIC data from this time is not reported. Images were taken every $5 \mathrm{~s}$ during the high-temperature hold.

The CR2 files from the camera were imported into the Adobe Photoshop Lightroom ${ }^{\circledR}$ software for editing. Images chosen as reference and distorted images were cropped, converted to grayscale, adjusted for brightness and contrast, adjusted for noise reduction, and exported as jpg files with lossless compression. Light room allows batch processing for this step. The extension LR/transporter was used to export the timestamp data for correlation with the synchrotron data files.

The speckle pattern on the edge of the sample is obscured due to the sample's curvature, and the shadow of the curved viewing hole obscures the corners of the cropped region. These reduce the usable area, so it is important to position the sample to maximize the size of the painted region in the field of view. The DIC software does not account for curvature so that unlike the longitudinal strain where real distance is considered, the transverse strain considers the projected distance. However, the width of the strain map is small compared to the specimen diameter, and therefore, the projected distance is no less than 0.98 of the real distance.

The open-source software $\mathrm{Ncorr}^{31}$ was used for the analysis. The final image taken during the $200{ }^{\circ} \mathrm{C}$ focusing hold was selected as the reference image, and the images taken during the hightemperature hold were selected as the distorted images. In the case of large displacement of the speckle pattern between images, in which a given reference speckle leaves the field of view, Ncorr's step analysis with automatic seed propagation feature was enabled, as recommended. ${ }^{31}$ During the displacement formatting step of Ncorr's analysis, the correlation coefficient cutoff was lowered to exclude regions of poor quality data caused by poor illumination. Resolution and pixel size information for the camera, cropped images, and strain map are listed in Table II. 
TABLE II. Resolution, pixel size, and related information.

\begin{tabular}{lc}
\hline \hline Camera & \\
\hline Pixel size (sensor) & $\begin{array}{c}4.29 \mu \mathrm{m} \\
\text { Sensor size }\end{array}$ \\
Sensor resolution & $22.3 \mathrm{~mm}^{2} \times 14.9 \mathrm{~mm}^{2}$ \\
\hline Cropped image & 5196 pixels $^{2} \times 3464$ pixels $^{2}$ \\
\hline Pixel size (object) & \\
Image size & $12 \mu \mathrm{m}$ \\
Image resolution & $3.7 \mathrm{~mm}^{2} \times 8.8 \mathrm{~mm}^{2}$ \\
Speckle size & 306 pixels ${ }^{2} \times 730$ pixels $^{2}$ \\
& $10-30$ pixels \\
\hline Displacement and strain & \\
\hline Subset radius & 48 pixels $(576 \mu \mathrm{m})$ \\
Subset spacing & 12 pixels $(144 \mu \mathrm{m})$ \\
Strain window radius & 2 pixels $(24 \mu \mathrm{m})$ \\
\hline \hline
\end{tabular}

The subset radius refers to the radius in pixels of each "unique local pattern," called a subset, identified by the software as they are distorted in subsequent images. The subset spacing refers to the center-to-center distance between each subset; the subset spacing should be one-fourth the radius. ${ }^{23}$ Strain is calculated using a leastsquares plane fit to a group of points in the displacement field called the strain window. The strain window radius acts as a smoothing parameter; the smallest strain radius that does not result in noisy data should be used. ${ }^{3}$

\section{E. Secondary validation experiment}

The material properties of SLM IN718 are not thoroughly known, so a test was carried out on a material with well-established properties to validate the accuracy of the computed DIC strains. 304 stainless steel was chosen for its high coefficient of linear thermal expansion (CTE or $\alpha$ ) and relatively high melting point. A flat bar of dimensions 12 in. $\times 1.5$ in. $\times 0.125$ in. was painted with hightemperature paint, as described earlier, and was secured vertically in the center of the heater. Its lower end was lightly clamped in place, with the upper end free to move. Two K-type thermocouples were secured to the sample, above and below the field of view. To obtain the largest field of view, the camera was positioned to acquire images through the beam entrance hole ( $0.75 \mathrm{in}$. diameter) rather than the 4-mm diameter viewing window that is used when collecting XRD data. The blue gel filter and macro-lens were used as described earlier. Three sets of DIC images were acquired, each under different heating conditions. The first was a ramp from $50^{\circ} \mathrm{C}$ to $300^{\circ} \mathrm{C}$ over $6 \mathrm{~min}$ or approximately $40^{\circ} \mathrm{C} / \mathrm{min}$. The second was a 4 -min hold at $300^{\circ} \mathrm{C}$; the measured strain should be zero during this hold. The third was a ramp from $570^{\circ} \mathrm{C}$ to $870^{\circ} \mathrm{C}$ over 3 min or approximately $100^{\circ} \mathrm{C} / \mathrm{min}$. Reported temperature values are the average of the temperatures reported by the two thermocouples. For each condition, DIC images were acquired every $10 \mathrm{~s}$.

\section{RESULTS AND DISCUSSION}

\section{A. Validation experiment results}

The results of the validation test were compared to theoretical strain based on the known CTE values of 304 stainless steel. ${ }^{32}$ The thermal strain, $\varepsilon_{\text {thermal }}$, was calculated from the CTE, here referred to as $\alpha$, using the following equation:

$$
\varepsilon_{\text {thermal }}=\alpha\left(T_{\text {final }}-T_{\text {initial }}\right) .
$$

The average temperature value reported by the two thermocouples was used as the temperature for calculations. CTE data for 304 stainless steel were extrapolated from manufacturer data ${ }^{32}$ using a linear fit with an $\mathrm{R}^{2}$ value of 0.966 .

The strains calculated by DIC and the theoretical strains for the third set of validation data are plotted in Fig. 6; only one set of data is presented for brevity. The DIC strains agree very well with the theoretical strains, indicating that the image quality is sufficient for DIC analysis. Scatter bars are the standard deviation of strain measurements of all pixels in a given strain map; the large scatter bars are in part indicative of variation in the strain field. Variation in the strain field may be indicative of inhomogeneous heating or texture. The error also increases with temperature as the lighting conditions become more difficult. (a)

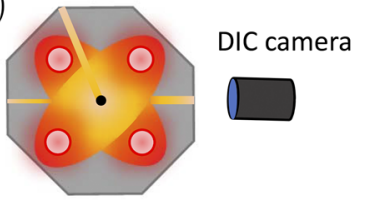

(b)

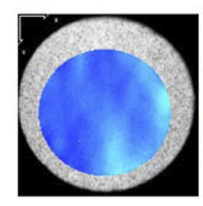

34 seconds

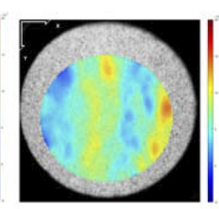

169 seconds (c)

DIC Strain vs. Theoretical Strain at High Temperature

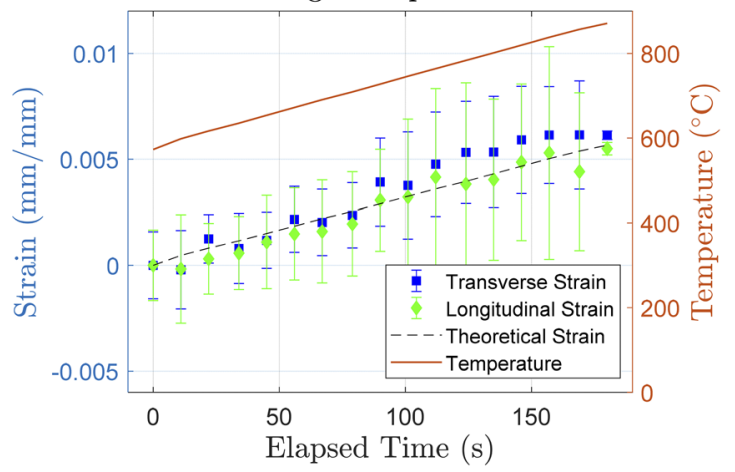

FIG. 6. Schematic of the camera position for the validation experiment on a flat bar of 304 stainless steel (a), sample strain fields at different elapsed time values during high-temperature ramp (b), and longitudinal and transverse DIC strains compared to theoretical strain based on CTE (c). The DIC strains are in good agreement with theoretical results, despite the difficulties of high high-temperature image capture with an enclosing heater. Statistical scatter bars represent standard deviation of measured strain values within a strain field as represented by pixel colors in Fig. 6(b). 

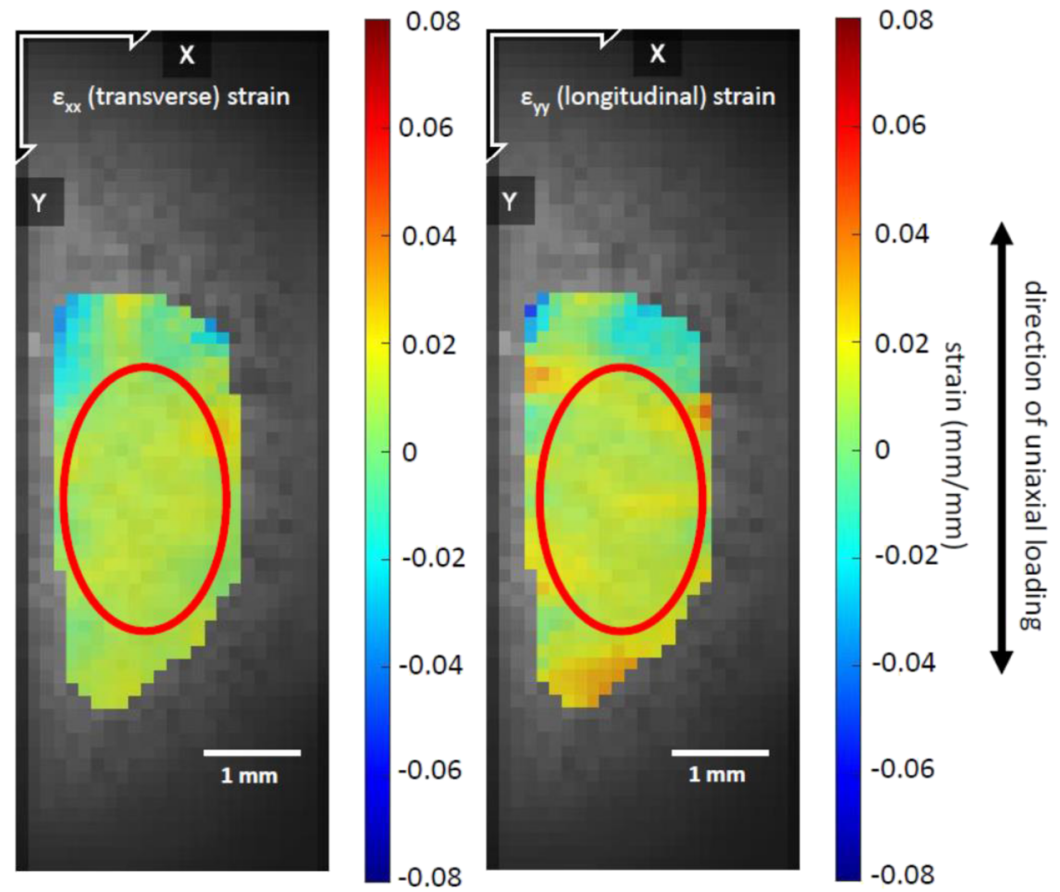

FIG. 7. Transverse $\left(\varepsilon_{x x}\right)$ and longitudinal $\left(\varepsilon_{y y}\right)$ strain maps of Inconel 718 after $47 \mathrm{~min}$ hold at $870^{\circ} \mathrm{C}$ and $0.98 \mathrm{kN}$ uniaxial tensile load. The red ellipses enclose the regions that are considered reliable and not affected by curvature, shadowing, or other artifacts.

\section{B. Primary experiment results}

The strain fields of the loaded IN718 sample at the end of the 47-min hold at $870^{\circ} \mathrm{C}$ and $0.98 \mathrm{kN}$ are shown in Fig. 7. The reference image was taken at $200^{\circ} \mathrm{C}$ with no tensile load. The strains in the direction of loading (longitudinal strain, $\varepsilon_{y y}$ ) and perpendicular to the direction of loading (transverse strain, $\varepsilon_{x x}$ ) are shown. The mean $\varepsilon_{y y}$ strain was 0.0099 , and the mean $\varepsilon_{x x}$ strain was 0.0066 . The asymmetric shape of the strain map was chosen to exclude regions where the speckle pattern is obscured by the curved shadow of the viewing hole, and the edge of the sample obscured by loss of focus due to sample curvature is also excluded.

The image was substantially cropped before DIC analysis; a comparison of a DIC strain field with the uncropped image is shown in Fig. 8, illustrating the challenge of obtaining sufficient images through a $4 \mathrm{~mm}$ window. The strains are expected to be homogeneous, consistent with uniaxial tensile load. Within the regions of the maps marked with red ellipses, the strains are relatively homogeneous; the regions outside these ellipses are affected by lower image quality from the difficult conditions.

Because the temperature and load were constant during the DIC strain measurements, the standard deviation of the strain measurements over time indicates the degree of noise in the strain calculation. This was $1.8 \times 10^{-3}$ and $1.5 \times 10^{-3}$ for the longitudinal and transverse strains, respectively. The noise is larger than usual for DIC, in great part due to lower quality of illumination. Having a larger viewing window and having the specimen centered within the viewing window would reduce the matching error in the displacement calculation. Spatial resolution would be improved by reducing the size of the paint speckles to the equivalent of 3 pixels, such as by using an air brush rather than an aerosol spray can.
The theoretical strain was calculated for comparison. The total longitudinal strain was calculated as the sum of mechanical strain (stress divided by Young's modulus at the temperature of loading), assuming only elastic behavior, and thermal strain [change in temperature multiplied by the coefficient of thermal expansion, CTE, as shown in Eq. (1)].

The average CTE values for temperature ranges from $25^{\circ} \mathrm{C}$ to a given temperature were taken from the IN718 data sheet and are reported in Table III. These values are the average CTE from $25^{\circ} \mathrm{C}$ to the indicated temperature $\mathrm{T}_{i}$, according to the following equation,

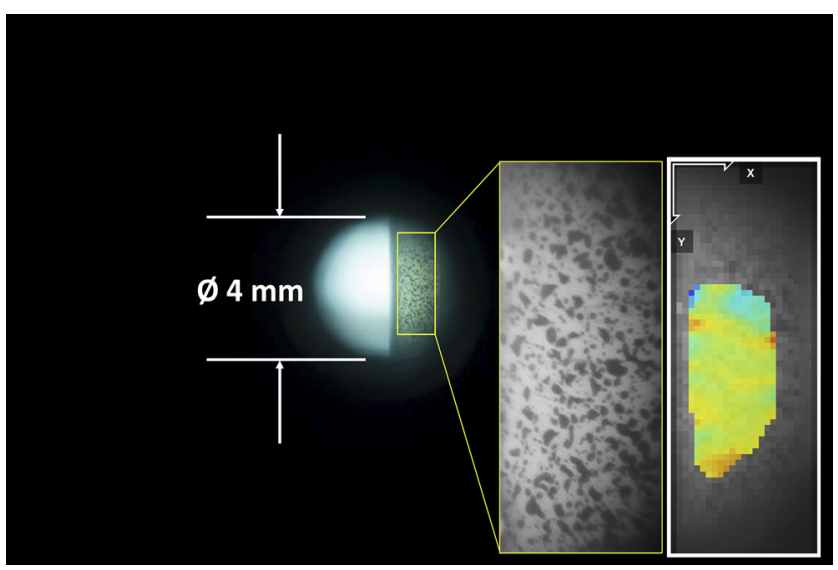

FIG. 8. Uncropped, unedited DIC image, with insets showing the edited cropped region and resulting strain field. 
TABLE III. CTE values for IN718, from $25^{\circ} \mathrm{C}$ to a given temperature. ${ }^{33}$

\begin{tabular}{lc}
\hline \hline Temperature $\left({ }^{\circ} \mathrm{C}\right)$ & $\mathrm{CTE}, 10^{-6} /{ }^{\circ} \mathrm{C}$ \\
\hline 93 & 12.8 \\
204 & 13.5 \\
316 & 13.9 \\
427 & 14.2 \\
538 & 14.4 \\
649 & 15.1 \\
760 & 16.0 \\
\hline \hline
\end{tabular}

where temperature is in Celsius:

$$
\alpha_{i}=\frac{\varepsilon_{i}}{T_{i}-25} .
$$

The CTE at $870^{\circ} \mathrm{C}$ was estimated to be $16.189 \times 10^{-6} /{ }^{\circ} \mathrm{C}$ from a linear extrapolation of the CTE data, with an $\mathrm{R}^{2}$ value of 0.958 . The thermal strain was calculated using Eq. (1), where $\alpha=16.189 \times 10^{-6}$, $T_{\text {final }}=870^{\circ} \mathrm{C}$, and $T_{\text {initial }}=200^{\circ} \mathrm{C}$.

Unlike conventionally cast material, SLM IN718 is anisotropic and is susceptible to higher porosity caused by local voids during deposition, contamination of the initial powder, and non-optimized process parameters. ${ }^{35}$ Young's modulus parallel to the build direction at room temperature is approximately $140 \mathrm{GPa},{ }^{36}$ and the temperature dependence of Young's modulus is assumed to be the same as for conventional IN718. Young's moduli of conventional IN718, shown in Table IV, were fit with a linear equation with an $\mathrm{R}^{2}$ value of 0.9988 . The slope of this equation was used with the data point of $\mathrm{E}=140 \mathrm{GPa}$ at $25^{\circ} \mathrm{C}$ to generate a linear equation for the temperature dependence of Young's modulus for SLM IN718. Using this equation, Young's modulus at $870^{\circ} \mathrm{C}$ was estimated to be $91.24 \mathrm{GPa}$. The mechanical strain was calculated according to the following equation, where $\mathrm{E}=91.24 \mathrm{GPa}$ :

$$
\varepsilon_{\text {mechanical }}=\frac{\sigma}{E} \text {. }
$$

The theoretical mechanical strain was calculated from this idealized isotropic model to be $5.487 \times 10^{-4}$ and the thermal strain to be $1.078 \times 10^{-2}$, for a total theoretical strain of 0.0113 in the direction of loading. This is $14 \%$ greater than the mean $\varepsilon_{y y}$ strain of 0.0099 from the DIC analysis, a good agreement considering the challenging conditions and the simplified model assumptions.

While the focus of this work is the DIC technique, a comparison between lattice and DIC strain data (shown in Fig. 9) is briefly

TABLE IV. Young's modulus values per temperature for conventional IN718. ${ }^{34}$

\begin{tabular}{lc}
\hline \hline Temperature $\left({ }^{\circ} \mathrm{C}\right)$ & $\mathrm{E}(\mathrm{GPa})$ \\
\hline 25 & 200 \\
200 & 191 \\
400 & 179 \\
600 & 167 \\
\hline \hline
\end{tabular}

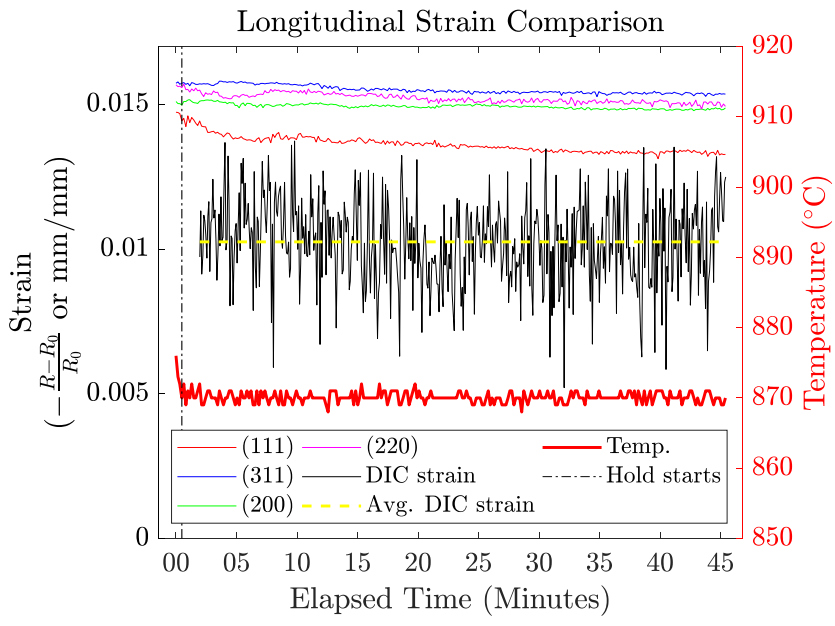

FIG. 9. While the DIC strain remains constant during the thermomechanical hold the longitudinal lattice strains show decreasing strain in the beginning of the hold, most dramatically in the (111) plane and least dramatically in the (311) and (200) planes. The DIC strain includes total strain, while the XRD strain only includes elastic strain.

discussed to provide an example of behavior that can be observed by combining DIC with simultaneous synchrotron measurements. There is a small difference in the strain-free reference used for the two techniques. The reference for the lattice strains was a sample at room temperature with no mechanical load. In contrast, the strains found using DIC use the position of surface speckles at $200^{\circ} \mathrm{C}$ and no mechanical load as the zero-strain reference positions. Additionally, the XRD measures only elastic strain, while the DIC measures both elastic and plastic strain. The inclusion of plastic strain in the DIC measurements implies that the measured DIC strain should be larger than or at least as large as the measured lattice strain. This is not the case for the strain data presented, which is largely due to using a higher temperature for the DIC zero-strain reference. Furthermore, in this experiment, the nominal stress remained below the yield stress, so no plastic strain occurred. Additionally, the sample's crystallographic texture caused uncertainty in determining the lattice strain. The texture resulted in spotty XRD diffraction rings, requiring interpolation to determine the strain-free reference radius. The sample's texture was confirmed by preparation of metallographic sections along the build direction and perpendicular to it, displaying columnar grains along the build direction. The longitudinal direction strains of four lattice planes are shown, each with a slightly different strain magnitude and behavior; these highlight the anisotropy in this material. For lattice strain measurements of FCC crystals in Ni-based alloys such as $\gamma$, it is recommended to compare the strain behavior of planes (111) and (311) to total strain behavior because they are weakly affected by intergranular strains. ${ }^{37}$ The (311) plane slowly relaxes in strain in the first $15 \mathrm{~min}$ of the hold by a small amount and then remains constant, while the (111) plane has a much more noticeable decrease in strain in the first $5 \mathrm{~min}$ of the hold, has smaller increase and decrease in strain up to $20 \mathrm{~min}$ into the hold, and then stabilizes. The (200) and (220) planes also exhibit fluctuations in strain in the first $5 \mathrm{~min}$ of the hold. As an 
additively manufactured material, the grain shape is expected to be similar to directionally solidified materials such that columnar grains are formed in the [001] direction. Coupled with results from DIC measurements, it is clear that changes in the microstructure occur and introduce variations in strain that are not observable on the macroscopic scale.

\section{CONCLUSIONS}

A method for using DIC to measure total strain with simultaneous XRD measurements at high temperature has been presented. This capability enables a real-time comparison between total strain and lattice strain under thermo-mechanical loading. These results demonstrate that this approach has the capability to non-destructively characterize the mechanical behavior of materials under extreme environments.

A preliminary experiment was conducted to validate the method. Measurements of the thermal strain in stainless steel 304 showed high accuracy for bi-axial strain information at high temperature. The method was applied to an XRD experiment to contrast lattice strains with the total strains. DIC results show the total strain state to be tensile and constant. Four lattice planes of the $\gamma$ matrix of the nickel alloy exhibited transient strain behavior in the beginning of the high-temperature hold, despite no observable change in the total strain, and these lattice planes had different strain magnitudes and behavior, highlighting the anisotropy of the strain response. As illustrated in Fig. 9, this method can be used to associate macroscopic and lattice behavior in real time. The method can also reveal inhomogeneous strain fields, if any, that may result from non-uniform temperature fields due to convection or sample misalignment.

The method presented is efficient and economical and can be applied to a variety of experimental conditions. The capability of performing DIC with an enclosing infrared heater expands opportunities for in situ data collection, and the fact that external lighting is not needed reduces the cost and complexity of the experimental setup. Collecting DIC images simultaneously with synchrotron $\mathrm{x}$-ray diffraction is a powerful tool to relate total and lattice strain in situ. DIC measurements can be combined with a variety of other non-destructive techniques, ${ }^{38}$ and this high-temperature application of DIC can be applied to many of them because it is low cost, portable, and simple to implement.

\section{ACKNOWLEDGMENTS}

This material is based on the work supported by the National Science Foundation (Grant Nos. DMR 1337758 and OISE 1460045) and by the German Science Foundation (DFG) (Grant No. SFBTRR 103, project A3). Use of the Advanced Photon Source, an Office of Science User Facility operated for the U.S. Department of Energy (DOE) Office of Science by Argonne National Laboratory, was supported by the U.S. DOE under Contract No. DE-AC02-06CH11357.

\section{REFERENCES}

${ }^{1}$ M. Sutton, W. Wolters, W. Peters, W. Ranson, and S. McNeill, Image Vision Comput. 1, 133 (1983).

${ }^{2}$ J. S. Lyons, J. Liu, and M. A. Sutton, Exp. Mech. 36, 64 (1996).

${ }^{3}$ B. Pan, K. Qian, H. Xie, and A. Asundi, Meas. Sci. Technol. 20, 062001 (2009).

${ }^{4}$ B. Pan, T. Jiang, and D. Wu, Rev. Sci. Instrum. 85, 115102 (2014).

${ }^{5}$ M. Anwander, B. G. Zagar, B. Weiss, and H. Weiss, Exp. Mech. 40, 98 (2000).

${ }^{6}$ V. Maurel, A. Köster, L. Rémy, M. Rambaudon, D. Missoum-Benziane, V. Fontanet, F. Salgado-Goncalves, A. Heudt, H. Wang, and M. Trabelsi, Int. J. Fatigue 102, 184 (2017).

${ }^{7}$ J. Blaber, B. S. Adair, and A. Antoniou, Rev. Sci. Instrum. 86, 035111 (2015).

${ }^{8}$ A. Chrysochoos, V. Huon, F. Jourdan, J.-M. Muracciole, R. Peyroux, and B. Wattrisse, Strain 46, 117 (2010).

${ }^{9}$ M. D. Novak and F. W. Zok, Rev. Sci. Instrum. 82, 115101 (2011).

${ }^{10}$ C. Bumgardner, B. Croom, and X. Li, Acta Mater. 128, 54 (2017).

${ }^{11}$ Y. Dong, H. Kakisawa, and Y. Kagawa, Opt. Lasers Eng. 68, 7 (2015).

${ }^{12}$ M. D. Strycker, L. Schueremans, W. V. Paepegem, and D. Debruyne, Opt. Lasers Eng. 48, 978 (2010).

${ }^{13}$ B. Pan, D. Wu, and Y. Xia, Opt. Lasers Eng. 48, 841 (2010).

${ }^{14}$ M. Harvey, C. Courcier, V. Maurel, and L. Rémy, in Proceedings of the 35th International Conference on Metallurgical Coatings and Thin Films [Surf. Coat. Technol. 203, 432 (2008)].

${ }^{15}$ B. Grant, H. Stone, P. Withers, and M. Preuss, J. Strain Anal. Eng. Des. 44, 263 (2009).

${ }^{16}$ B. Pan, D. Wu, Z. Wang, and Y. Xia, Meas. Sci. Technol. 22, 015701 (2010).

${ }^{17}$ X. Chen, N. Xu, L. Yang, and D. Xiang, Meas. Sci. Technol. 23, 125603 (2012).

${ }^{18}$ X. Guo, J. Liang, Z. Tang, B. Cao, and M. Yu, Opt. Eng. 53, 063101 (2014).

${ }^{19}$ R. B. Berke and J. Lambros, Rev. Sci. Instrum. 85, 045121 (2014).

${ }^{20}$ B. Pan, D. Wu, and J. Gao, J. Strain Anal. Eng. Des. 49, 224 (2014).

${ }^{21}$ P. Meyer and A. Waas, Exp. Mech. 55, 795 (2015).

${ }^{22}$ W. Wang, C. Xu, H. Jin, S. Meng, Y. Zhang, and W. Xie, Meas. Sci. Technol. 28, 035007 (2017).

${ }^{23}$ G. Lionello and L. Cristofolini, Meas. Sci. Technol. 25, 107001 (2014).

${ }^{24}$ S. F. Siddiqui, K. Knipe, A. Manero, C. Meid, J. Wischek, J. Okasinski, J. Almer, A. M. Karlsson, M. Bartsch, and S. Raghavan, Rev. Sci. Instrum. 84, 083904 (2013).

${ }^{25}$ K. Knipe, A. C. Manero, S. Sofronsky, J. Okasinski, J. Almer, J. Wischek, C. Meid, A. Karlsson, M. Bartsch, and S. Raghavan, J. Eng. Gas Turbines Power 137, 082506 (2015).

${ }^{26}$ D. Haeffner, J. Almer, and U. Lienert, Mater. Sci. Eng.: A 399, 120 (2005).

${ }^{27}$ B. B. He, U. Preckwinkel, and K. L. Smith, Adv. X-ray Anal. 42, 429 (2000).

${ }^{28}$ K. Knipe, A. Manero II, S. F. Siddiqui, C. Meid, J. Wischek, J. Okasinski, J. Almer, A. M. Karlsson, M. Bartsch, and S. Raghavan, Nat. Commun. 5, 4559 (2014).

${ }^{29}$ A. Manero, K. Knipe, J. Wischek, C. Meid, J. Okasinski, J. Almer, A. Karlsson, M. Bartsch, and S. Raghavan, Coatings 8, 320 (2018).

${ }^{30} \mathrm{~T}$. Armes, Lr/transporter, 2015.

${ }^{31}$ J. Blaber, B. Adair, and A. Antoniou, Exp. Mech. 55, 1105 (2015).

${ }^{32}$ 304/304l stainless steel data sheet, AK Steel, 2007.

${ }^{33}$ High Temp Metals, Inconel 718 technical data, 2015.

${ }^{34}$ Alloy 718, Lamineries Matthey SA data sheet, 2013.

${ }^{35}$ E. Chlebus, K. Gruber, B. Kunicka, J. Kurzac, and T. Kurzynowski, Mater. Sci. Eng.: A 639, 647 (2015).

${ }^{36} \mathrm{M}$. Bartsch, Institute of Materials Research (German Aerospace Center, Cologne, 2017).

${ }^{37}$ M. T. Hutchings, P. J. Withers, T. M. Holden, and T. Lorentzen, Introduction to the Characterization of Residual Stress by Neutron Diffraction (CRC Press, 2005).

${ }^{38}$ G. Freihofer, A. Schülzgen, and S. Raghavan, NDT \&E Int. 75, 65 (2015). 